\title{
A new notion of transitivity for groups and sets of permutations
}

\author{
William J. Martin and Bruce E. Sagan \\ 1 Department of Mathematical Sciences \\ and Department of Computer Science \\ Worcester Polytechnic Institute \\ martin@wpi.edu \\ 2 Department of Mathematics \\ Michigan State University \\ sagan@math.msu.edu
}

\begin{abstract}
Let $\Omega=\{1,2, \ldots, n\}$ where $n \geq 2$. The shape of an ordered set partition $P=\left(P_{1}, \ldots, P_{k}\right)$ of $\Omega$ is the integer partition $\lambda=$ $\left(\lambda_{1}, \ldots, \lambda_{k}\right)$ defined by $\lambda_{i}=\left|P_{i}\right|$. Let $G$ be a group of permutations acting on $\Omega$. For a fixed partition $\lambda$ of $n$, we say that $G$ is $\lambda$-transitive if $G$ has only one orbit when acting on partitions $P$ of shape $\lambda$. A corresponding definition can also be given when $G$ is just a set. For example, if $\lambda=(n-t, 1, \ldots, 1)$, then a $\lambda$-transitive group is the same as a $t$ transitive permutation group and if $\lambda=(n-t, t)$, then we recover the $t$-homogeneous permutation groups.

In this paper, we use the character theory of the symmetric group $S_{n}$ to establish some structural results regarding $\lambda$-transitive groups and sets. In particular, we are able to generalize a theorem of Livingstone and Wagner [12] about $t$-homogeneous groups. We survey the relevant examples coming from groups. While it is known that a finite group of permutations can be at most 5-transitive unless it contains the alternating group, we show that it is possible to construct a non-trivial $t$-transitive set of permutations for each positive integer $t$. We also show how these ideas lead to a split basis for the association scheme of the symmetric group.
\end{abstract}

\section{Introduction}

Throughout, let $n \geq 2$ be an integer and let $\Omega=\{1, \ldots, n\}$. Suppose that $G$ is a subgroup of the symmetric group $S_{n}$. Recall that $G$ is $t$-transitive if it is transitive on ordered $t$-tuples of distinct elements of $\Omega$. It is trivial to see that if $G$ is $t$-transitive, then it is $(t-1)$-transitive. Similarly, $G$ is $t$-homogeneous if it is transitive on $t$-element subsets of $\Omega$. The following is one of the main theorems in a paper of Livingstone and Wagner.

Theorem 1 ([12]). Let $G \leq S_{n}$ be $t$-homogeneous where $t \leq n / 2$. Then $G$ is also $(t-1)$-homogeneous. 
Our goal is to define a more general notion of transitivity so that both the $t$-transitive and $t$-homogeneous results will be special cases. To this end, let an ordered set partition of $\Omega$ be an ordered tuple

$$
P=\left(P_{1}, \ldots, P_{k}\right)
$$

of pairwise disjoint non-empty subsets of $\Omega$ whose union is $\Omega$. These partitions are also called tabloids and play an important role in the representation theory of $S_{n}$ [9 14. We will only need to consider the case where $\left|P_{i}\right| \geq\left|P_{i+1}\right|$ for all $1 \leq i<k$. On the other hand, an (integer) partition $\lambda$ of $n$, written $|\lambda|=n$, is a sequence

$$
\lambda=\left(\lambda_{1}, \ldots, \lambda_{k}\right)
$$

of positive integers whose sum is $n$ and satisfying the monotonicity condition

$$
\lambda_{1} \geq \lambda_{2} \geq \cdots .
$$

The $\lambda_{i}$ are called the parts of $\lambda$. The shape of the set partition $P$ is the integer partition

$$
|P|=\left(\left|P_{1}\right|, \ldots,\left|P_{k}\right|\right) .
$$

Let $\lambda$ be a fixed partition of $n$. A subgroup $G$ of $S_{n}:=\operatorname{Sym}(\Omega)$ is said to be $\lambda$-transitive if it is transitive on partitions $P$ of shape $\lambda$. Equivalently, for any two set partitions $P$ and $Q$ with $|P|=|Q|=\lambda$, there exists $g \in G$ taking $P$ to $Q$ in the sense that

$$
g P_{i}=Q_{i}
$$

(as a set) for each $i, 1 \leq i \leq k$. This definition includes that of a $t$-transitive permutation group (use $\lambda=(n-t, 1, \ldots, 1)$ ) and that of a $t$-homogeneous permutation group (use $\lambda=(n-t, t)$ ).

We extend this definition to arbitrary sets of permutations as follows. For $D \subseteq S_{n}$ and $\lambda$ a partition of $n$, we say that $D$ is $\lambda$-transitive if there is a constant $r_{\lambda}$ such that, for any two set partitions $P$ and $Q$ of shape $\lambda, D$ contains exactly $r_{\lambda}$ permutations $g$ taking $P$ to $Q$. We will also use the terminology " $t$ transitive," respectively "t-homogeneous," for sets of permutations which are $(n-t, 1, \ldots, 1)$-transitive, respectively $(n-t, t)$-transitive.

The following two propositions follow directly from the definitions.

Proposition 1. If $G \leq S_{n}$ is a $\lambda$-transitive group of permutations acting on $\Omega_{n}$, then $G$ is also $\lambda$-transitive as a set.

Proposition 2. If $D \subseteq S_{n}$ is a $\lambda$-transitive set of permutations acting on $\Omega_{n}$ and $G=\langle D\rangle$ is the subgroup generated by $D$, then $G$ is a $\lambda$-transitive group.

Each set partition $P$ determines a Young subgroup $Y=Y_{P}$ which consists of the set of permutations of $\Omega$ which fix each subset $P_{i}$ of $P$ setwise. We say $Y$ has shape $\lambda$ where $\lambda=|P|$. There is a bijection between all set partitions of shape $\lambda$ and all left cosets of $Y_{P}$ that preserves the action of $S_{n}$. Using this observation, it is easy to prove the following characterization of $\lambda$-transitivity. 
Lemma 1. Suppose $|\lambda|=n$ and $D \subseteq S_{n}$. Then $D$ is $\lambda$-transitive if and only if there is a constant $r_{\lambda}$ such that for any coset $g Y_{P}$ of any Young subgroup of shape $\lambda$ we have $\left|g Y_{P} \cap D\right|=r_{\lambda}$.

The last definition that will be necessary to understand our generalization of the Livingstone-Wagner Theorem and ( $t$-transitivity result) is that of the dominance order on integer partitions. If $|\lambda|=|\mu|=n$ then $\lambda$ is dominated by $\mu$, written $\lambda \unlhd \mu$, if and only if

$$
\sum_{i=1}^{j} \lambda_{i} \leq \sum_{i=1}^{j} \mu_{i} \quad \text { (for all } j \text { ) }
$$

where we define $\lambda_{i}=0$ if $i$ is greater than the number of parts in $\lambda$ and similarly for $\mu$. We will give two proofs that if $\mu \unrhd \lambda$ then $\lambda$-transitivity implies $\mu$-transitivity, from which Theorem 1 follows immediately. The first, which is just for groups but also includes a stronger result about orbits, is essentially a generalization of the one given by Livingstone and Wagner themselves. The second proof holds for all $\lambda$-transitive sets $D$. But if $D$ is not a group then it does not have orbits and so a combinatorial approach is needed in this case.

\section{The group case}

Let the permutation group $G$ act on two sets $S$ and $T$ with characters $\chi$ and $\psi$, respectively. The inequality $\chi \geq \psi$ means that $\chi=\psi+\phi$ where $\phi$ is some character of $G$. Equivalently, if $\chi^{1}, \ldots, \chi^{k}$ are the irreducible characters of $G$ and we write $\chi=\sum_{i} c_{i} \chi^{i}, \psi=\sum_{i} d_{i} \chi^{i}$ for nonnegative integers $c_{i}, d_{i}$ then $\chi \geq \psi$ if and only if $c_{i} \geq d_{i}$ for all $i$. We will also need the notation

$$
\operatorname{orb}(G, S)=\text { the number of orbits of } G \text { acting on } S \text {. }
$$

Lemma 2. Let $G$ be a permutation group acting on sets $S$ and $T$ with characters $\chi$ and $\psi$, respectively. If $\chi \geq \psi$ then

$$
\operatorname{orb}(G, S) \geq \operatorname{orb}(G, T) .
$$

Proof. Taking inner products with the trivial character and using $\chi=\psi+\phi$, we have

$$
\operatorname{orb}(G, S)=\langle\chi, 1\rangle=\langle\psi+\phi, 1\rangle=\langle\psi, 1\rangle+\langle\phi, 1\rangle \geq\langle\psi, 1\rangle=\operatorname{orb}(G, T) . \square
$$

We now apply this lemma to the situation at hand. Let $P$ be any set partition of shape $\lambda$ and let $S_{\lambda}$ denote the set of all left cosets of the Young subgroup $Y_{P}$. Of course, $S_{\lambda}$ depends on $P$ and not just $\lambda$, but that fact will not play a role in the following theorem.

Theorem 2. If $G \leq S_{n}$ then

$$
\lambda \unlhd \mu \Longrightarrow \operatorname{orb}\left(G, S_{\lambda}\right) \geq \operatorname{orb}\left(G, S_{\mu}\right) .
$$


Proof. Let $\chi$ and $\psi$ be the characters of $G$ acting on $S_{\lambda}$ and $S_{\mu}$, respectively. Then by the previous lemma, it suffices to show that $\chi \geq \mu$. Let $P$ and $Q$ be ordered set partitions giving rise to the sets $S_{\lambda}$ and $S_{\mu}$, respectively. Then $\chi$ and $\psi$ are the restrictions to $G$ of the characters $\chi^{\prime}=1_{S_{\lambda}} \uparrow^{S_{n}}$ and $\psi^{\prime}=1_{S_{\mu}} \uparrow S_{n}$, respectively, so we are reduced to showing $\chi^{\prime} \geq \psi^{\prime}$. But then by Young's Rule

$$
\chi^{\prime}=\sum_{\nu \unrhd \lambda} K_{\nu \lambda} \chi^{\nu} \quad \text { and } \quad \psi^{\prime}=\sum_{\nu \unrhd \mu} K_{\nu \mu} \chi^{\nu}
$$

where $\chi^{\nu}$ is the irreducible character of $S_{n}$ corresponding to $\nu$ and $K_{\nu \lambda}$ is a Kostka number. However, it is known [11, 18 that if $\lambda \unlhd \mu$ then $K_{\nu \lambda} \geq K_{\nu \mu}$ for all $\nu$. This finishes the proof.

As an immediate corollary, we get our first generalization of the LivingstoneWagner Theorem.

Theorem 3. If $G \leq S_{n}$ is $\lambda$-transitive and $\mu \unrhd \lambda$ then $G$ is $\mu$-transitive.

\section{The center of the group algebra and the inner distribution}

Our main tool for extracting information about $\lambda$-transitive sets will be the center of the group algebra of the symmetric group $S_{n}$, specifically in the form arising from the left regular representation $g \mapsto A(g)$ (over the reals). We will denote this commutative algebra of symmetric matrices by $\mathbb{A}$ and its dimension by $p(n)$, the number of partitions of the integer $n$. One may learn quite a bit about this algebra via the character theory of the symmetric group; see references [1,9414. We identify two distinguished bases of $\mathbb{A}$ as follows. The first basis contains one matrix for each conjugacy class $\mathcal{C}_{\alpha}$, namely

$$
A_{\alpha}=\sum_{g \in \mathcal{C}_{\alpha}} A(g) .
$$

The second distinguished basis is the basis of primitive idempotents for the center. This basis contains one element, $E_{\mu}$ say, for each irreducible representation of $S_{n}$. The two bases are related by the equations

$$
E_{\mu}=\frac{f_{\mu}}{n !} \sum_{\alpha} \chi_{\alpha}^{\mu} A_{\alpha}
$$

where $f_{\mu}$ is the degree of the irreducible representation $\rho_{\mu}$ and $\chi_{\alpha}^{\mu}$ is the value taken by the corresponding irreducible character on the conjugacy class $\mathcal{C}_{\alpha}$ [1, Theorem II.7.2].

The matrices in $\mathbb{A}$ are clearly simultaneously diagonalizable. The eigenspace of $\mathbb{A}$ indexed by $\mu$ is the column space of $E_{\mu}$ :

$$
V_{\mu}=\operatorname{colsp}\left(E_{\mu}\right)
$$

The dimension of $V_{\mu}$ is $f_{\mu}^{2}$. 
Let $D$ be a non-empty subset of $S_{n}$ and let $x=x_{D}$ denote the indicator vector of $D$. This is a 01-vector of length $n$ ! with $x(g)=1$ if and only if $g \in D$. The inner distribution of $D$ is the rational vector of length $p(n)$ with $\alpha$ entry equal to

$$
a_{\alpha}=\frac{1}{|D|} x^{\top} A_{\alpha} x
$$

The dual distribution of $D$ is the vector $b$ with entries indexed by irreducible representations and defined by

$$
b_{\mu}=\frac{n !}{|D|} x^{\top} E_{\mu} x
$$

Since each $E_{\mu}$ is positive semi-definite, we have $b \geq 0$ and $b_{\mu}=0$ if and only if $E_{\mu} x=0$. And since $E_{\mu}$ is the matrix representing orthogonal projection onto $V_{\mu}$ we have another equivalent condition, namely $b_{\mu}=0$ if and only if $x \perp V_{\mu}$.

For arbitrary $D$, the quantities $a_{\alpha}$ and $b_{\mu}$ have alternative expressions as follows:

$$
a_{\alpha}=\frac{1}{|D|}\left|\left\{(g, h) \in D \times D: g h^{-1} \in \mathcal{C}_{\alpha}\right\}\right| ;
$$

and

$$
b_{\mu}=\frac{f_{\mu}}{|D|} \sum_{g, h \in D} \chi^{\mu}\left(g h^{-1}\right) .
$$

When $D$ is a group, these simplify to

$$
a_{\alpha}=\left|D \cap \mathcal{C}_{\alpha}\right|
$$

and

$$
b_{\mu}=f_{\mu} \sum_{g \in D} \chi^{\mu}(g)
$$

\section{The set case}

Lemma 3. Suppose $D$ is a coset of a Young subgroup of shape $\lambda$ and $b$ is the dual distribution of $D$. Then $b_{\mu} \neq 0$ if and only if $\mu \unrhd \lambda$.

Proof. Because of equation (2), we may assume $D$ is a Young subgroup. In this case, by Young's rule and (3), the number $b_{\mu}$ is a non-zero multiple of the Kostka number $K_{\mu \lambda}$.

Let $Y_{\lambda}$ denote the incidence matrix of $S_{n}$ versus cosets of Young subgroups of shape $\lambda$. The $n$ ! rows of $Y_{\lambda}$ are indexed by permutations and the columns are indexed by all left cosets $h Y_{P}$ of all Young subgroups of shape $\lambda$. In the entry indexed $\left(g, h Y_{P}\right)$, we have a 1 if $g \in h Y_{P}$ and a 0 otherwise.

Corollary 1. Let $W_{\lambda}$ be the column space of $Y_{\lambda}$. Then

$$
W_{\lambda}=\bigoplus_{\mu \unrhd \lambda} V_{\mu}
$$


Proof. This space is invariant under the left action of the group algebra. So, in particular, it is $\mathbb{A}$ invariant and thus a sum of eigenspaces. Combining this with the previous lemma yields the desired result.

Theorem 4. Let $D$ be a set of permutations in $S_{n}$ and $\lambda$ a partition of $n$. Then $D$ is $\lambda$-transitive if and only if for every $\mu$ with $\lambda \unlhd \mu \triangleleft(n)$ we have $b_{\mu}=0$.

Proof. We prove the forward implication noting that the converse follows by reversing the steps of the proof.

¿From Corollary 1, the eigenspaces which appear in $W_{\lambda}$ are precisely those $V_{\mu}$ for which $\mu \unrhd \lambda$. Now if $x$ denotes the indicator vector of $D$, then by Lemma 1 we have $x^{\top} Y_{\lambda}=r_{\lambda} \mathbf{1}$ where $\mathbf{1}$ stands for the all ones vector of appropriate length. Let $x_{0}$ denote the projection of $x$ onto $W_{\lambda}$. Note that $x$, and thus $x_{0}$, has the same inner products with columns of $Y_{\lambda}$ as does the vector $\frac{r_{\lambda}}{\left|Y_{P}\right|} \mathbf{1}$ where $P$ is of shape $\lambda$. So the same will be true for products with the vectors from any orthonormal basis $B$ for $W_{\lambda}$. It follows that $x_{0}$ is equal to a multiple of the all ones vector. Since $x_{0} \in V_{(n)}$, we have $x \perp V_{\mu}$ for all $\mu$ such that $\lambda \unlhd \mu \triangleleft(n)$ and hence the theorem.

An immediate corollary of this result is our second generalization of the Livingstone-Wagner Theorem.

Theorem 5. If the set $D$ is $\lambda$-transitive and $\mu \unrhd \lambda$, then $D$ is $\mu$-transitive.

We get a second corollary of Theorem 4 by combining it with equation (2).

Theorem 6. The set $D$ is $\lambda$-transitive if and only if

$$
\sum_{g, h \in G} \chi^{\mu}\left(g h^{-1}\right)=0
$$

for each $\mu$ such that $\lambda \unlhd \mu \triangleleft(n)$.

\section{Examples}

¿From Theorem 3, we know that a $\lambda$-transitive group of permutations is $t$ homogeneous where $t=\sum_{i=2}^{k} \lambda_{i}$. Thus we are severely restricted in our search for $\lambda$-transitive groups. Other than alternating and symmetric groups, there are no $t$-homogeneous groups for $t \geq 6$. (This is a consequence of the classification of finite simple groups; see [7] or [3].) There are precisely two non-trivial 5 -transitive groups: the Mathieu groups $M_{12}$ and $M_{24}$. Of course, a $t$-transitive group is $\lambda$-transitive for any partition $\lambda$ refined by $(n-t, 1, \ldots, 1)$. So we seek $t$-homogeneous groups which fail to be $t$-transitive. Livingstone and Wagner [12] showed that there are no such groups for $t \geq 5$. The balance of the following result is due to Kantor [10] who dealt with the cases $t=2,3,4$.

Theorem 7 (Kantor, Livingstone/Wagner [7]). Suppose $G$ is a permutation group acting $t$-homogeneously on $n$ points $(t \leq n / 2)$, but not $t$-transitively. Then one of the following is the case: 
- $t=2, A S L_{1}(q) \leq G \leq A \Sigma L_{1}(q), q \equiv 3 \bmod 4$;

- $t=3, P S L_{2}(q) \leq G \leq P \Sigma L_{2}(q), q \equiv 3 \bmod 4$;

- $t=3, G=A G L_{1}(8), A \Gamma L_{1}(8), A \Gamma L_{1}(32)$;

- $t=4, G=P G L_{2}(8), P \Gamma L_{2}(8), P \Gamma L_{2}(32)$.

In all cases, the group acts $(t-1)$-transitively.

For each group appearing in this theorem, we wish to determine its full level of transitivity. (For $t=2$, there is no partition between $(n-2,2)$ and $(n-2,1,1)$ to consider.)

- For $P S L_{2}(q)$ acting on the projective line $(q+1$ points $)$, we find the stabilizer of a 3 -set has size three. Since the action is faithful, we conclude that $P S L_{2}(q)$ acts $(n-3,2,1)$-transitively on the projective line. It follows that this is also the full degree of transitivity of $P \Sigma L_{2}(q)$ and any group between the two.

- $A G L_{1}(8)$ has order 56 , so this group acts sharply 3 -homogeneously on $\mathbb{F}_{8}$.

- $A \Gamma L_{1}(8)$ acts faithfully on $\mathbb{F}_{8}$ with three group elements stabilizing any 3 -set. So this action is $(5,2,1)$-transitive.

- $A \Gamma L_{1}(32)$ has order 4960 , so this group acts sharply 3-homogeneously on $\mathbb{F}_{32}$.

- Finally, we consider the groups with $t=4$. First, $P G L_{2}(8)$ has 504 elements and acts faithfully on $P G(1,8)$ with the stabilizer of any 4 -set being isomorphic to the Klein four-group, so it is $(5,3,1)$-transitive in this action.

- $P \Gamma L_{2}(8)$ has 1512 elements and acts 4-homogeneously on nine points. The stabilizer of a 4 -set has size 12 , and thus is isomorphic to $A_{4}$. Hence this group is $(5,2,1,1)$-transitive.

- For $P \Gamma L_{2}(32)$, the stabilizer of a 4 -set is isomorphic to the Klein four-group, so the action is $(29,3,1)$-transitive.

We note that, in 1975, Peter Neumann [13] gave a classification scheme for $t$ homogeneous groups which are not fully $t$-transitive in terms of the isomorphism type of the stabilizer of a $t$-set. In Neumann's language, a group which acts almost generously 3-transitively is one in which the stabilizer of any 4-set contains $A_{4}$. (He does not presume a 4-homogeneous action in this definition.) Clearly a group acting 4-homogeneously has this property exactly when it is $(n-4,2,1,1)$ transitive. Neumann calls a permutation group a little generously 3 -transitive if the stabilizer of any 4-set contains the Klein four-group. Observe that a group acting 4-homogeneously and having this property will be $(n-4,3,1)$-transitive. Since there are no 4-homogeneous groups with cyclic stabilizer of a 4 -set, the converse is true as well. But this observation hinges on Kantor's result.

We now construct a family of $\lambda$-transitive sets of permutations for partitions $\lambda$ having the property that any $\lambda$-transitive group must be strictly larger than the set we construct.

Example 1. [2] For $q$ an odd prime power, the affine group $A G L_{1}(q)$ acts 2transitively on $\mathbb{F}_{q}$. There can be no 2-homogeneous group of size $\left(\begin{array}{l}q \\ 2\end{array}\right)$ but there is 
such a set of permutations. Choose a subset $S$ of $\mathbb{F}_{q}-\{0\}$ such that $S$ is disjoint from $-S$ and $S \cup(-S)=\mathbb{F}_{q}-\{0\}$. (Since $q$ is odd, this is always possible.) Let

$$
D=\left\{x \mapsto a x+b: a \in S, b \in \mathbb{F}_{q}\right\} .
$$

We claim that this set is $(q-2,2)$-transitive. For if the mapping $x \mapsto a x+b$ takes $u$ to $y$ and $v$ to $z$, then the mapping which takes $u$ to $z$ and $v$ to $y$ (in $\left.A G L_{1}(q)\right)$ is $x \mapsto(-a) x+b^{\prime}$ for some $b^{\prime}$. Thus no two elements of $D$ take an unordered pair $\{u, v\}$ to the same image.

\section{A recursive construction}

In this section, we show that, given any partition $\lambda$ of a positive integer, $m$ say, if we are permitted to increase the largest part (thus obtaining a partition $\lambda^{\prime}$ of some integer $n \geq m$ ), we can construct a $\lambda^{\prime}$-transitive set of permutations $D$ which is "small" in comparison to $\left|S_{n}\right|$.

Suppose the following three structures are given:

- a $t-(n, k, \nu)$ block design on point set $\Omega=\{1, \ldots, n\}$ with block set $B$;

- a $t$-transitive set of permutations $D_{1} \subseteq S_{k}$;

- a $t$-transitive set of permutations $D_{2} \subseteq S_{n-k}$.

¿From these, we will construct a $t$-transitive set $D$ of permutations inside $S_{n}$.

We first define a number of bijections. Let

$$
\tau:\{k+1, \ldots, n\} \longrightarrow\{1, \ldots, n-k\}
$$

via $j \mapsto j-k$. For each block $b \in B$, choose bijections

$$
\phi_{b}:\{1, \ldots, k\} \longrightarrow b
$$

and

$$
\psi_{b}:\{1, \ldots, n-k\} \longrightarrow \Omega-b,
$$

which will remain fixed.

Lemma 4. With the above notation, the set of functions

$$
D=\left\{\left(\phi_{b} \circ \pi\right) \cup\left(\psi_{b} \circ \sigma \circ \tau\right): b \in B, \pi \in D_{1}, \sigma \in D_{2}\right\}
$$

is a $t$-transitive set of permutations in $S_{n}$.

Example 2. Suppose $t=2, n=7, k=3$ and the blocks are those of the Fano plane

$$
B=\{124,235,346,457,561,672,713\},
$$

$D_{1}=S_{3}$ and $D_{2}=A_{4}$. We then obtain a 2 -transitive set of $7.6 .12=504$ permutations in $S_{7}$. For instance, if $b=\{3,4,6\}$ and $\Omega-b=\{5,7,1,2\}$ (listed in the order induced by $\phi_{b}$ and $\left.\psi_{b}\right)$ and $\pi=(12), \sigma=(13)(24)$, the resulting permutation in $D$ is

$$
\left(\phi_{b} \circ \pi\right) \cup\left(\psi_{b} \circ \sigma \circ \tau\right)=(14)(2365) .
$$


The following notation will be required in the proof of this lemma. Suppose $I$ and $O$ are disjoint subsets of $\Omega$. We would like to count blocks $b \in B$ which contain all of the points in $I$ yet none of the points in $O$. If $|I|=i$ and $|O|=j$ and $i+j \leq t$, then there exists a constant $\nu_{i, j}$ such that there exactly $\nu_{i, j}$ blocks $b$ such that $I \subseteq b$ and $O \cap b=\emptyset[3$, Remark 4.17].

Suppose that $h \leq t$. If $a_{1}, \ldots, a_{h}$ are distinct elements of $\Omega_{k}$ and $b_{1}, \ldots, b_{h}$ are distinct elements of $\Omega_{k}$, then there are exactly $c_{h}$ permutations $\pi \in D_{1}$ such that $\pi a_{j}=b_{j}$ for all $1 \leq j \leq h$ for some constant $c_{h}$. Similarly, there exist constants $d_{0}, \ldots, d_{t}$ associated to $D_{2}$.

Proof of Lemma 5. Let $a_{1}<\cdots<a_{t}$ be distinct elements of $\Omega$ and let $b_{1}, \ldots, b_{t}$ be distinct elements of $\Omega$. If $a_{1}>k$, set $i=0$; otherwise, let $i$ be the largest integer such that $a_{i} \leq k$ and set $j=t-i$. There are $\nu_{i, j}$ blocks $b$ of the design $(\Omega, B)$ for which $b_{1}, \ldots, b_{i} \in b$ and $b_{i+1}, \ldots, b_{t} \notin b$. There are $c_{i}$ permutations $\pi \in D_{1}$ taking $a_{\ell}$ to $\phi_{b}^{-1} b_{\ell}$ for $\ell \leq i$. There are $d_{j}$ permutations $\sigma$ in $D_{2}$ taking $a_{\ell}-k$ to $\psi_{b}^{-1} b_{\ell}$ for $i<\ell \leq t$. Thus there are

$$
r=c_{i} d_{j} \nu_{i, j}
$$

permutations $\rho \in D$ satisfying $\rho a_{\ell}=b_{\ell}$ for all $1 \leq \ell \leq t$. To finish the proof, we simply must show that this quantity $r$ does not depend on $i$. But we have

$$
\nu_{i+1, j}(n-i-j)=\nu_{i, j}(k-i)
$$

and

$$
\nu_{i, j+1}(n-i-j)=\nu_{i, j}(n-k-j)
$$

giving us

$$
\nu_{i+1, j-1}(n-k-j+1)=\nu_{i, j}(k-i) .
$$

Moreover, it is obvious that $c_{i}=c_{i+1}(k-i)$ and $d_{j-1}=d_{j}(n-k-j+1)$. Putting these identities together, we find that

$$
\nu_{i, j} c_{i} d_{j}=\nu_{i+1, j-1} c_{i+1} d_{j-1}
$$

which completes our proof.

We will now make use of the following result:

Theorem 8 (Teirlinck 15). Given integers $t$ and $n$ with

$$
n \equiv t \quad\left(\bmod (t+1) !^{2 t+1}\right)
$$

and $n \geq t+1>0$, there exists a $t-\left(n, t+1,(t+1) !^{2 t+1}\right)$ design without repeated blocks.

Using these designs in the previous construction, we obtain the following asymptotic existence theorem.

Theorem 9. Let $\lambda_{2} \geq \lambda_{3} \geq \cdots \geq \lambda_{t}$ be a non-increasing sequence of positive integers and let $\epsilon>0$. Then there exist infinitely many values for $\lambda_{1} \geq \lambda_{2}$ such that there exists a $\lambda$-transitive set $D$ of permutations inside $S_{n}\left(n=\lambda_{1}+\cdots+\lambda_{t}\right)$ having $|D| / n !<\epsilon$. 
Proof. It suffices to prove this for $\lambda_{2}=\cdots=\lambda_{t}=1$. Let $k=t+1$ and choose $n \geq 2 t+1$ to satisfy Teirlinck's theorem. Then there exists a block design $B$ on $n$ points with each $t$-set appearing in exactly $(t+1) !^{2 t+1}$ blocks. Using $S_{t+1}$ and $S_{n-t-1}$ as our component $t$-transitive permutation sets, we obtain a $t$-transitive set $D$ of permutations in $S_{n}$ with

$$
|D|=|B| \cdot(t+1) ! \cdot(n-t-1) !=\frac{(t+1) !^{2 t+1}}{t+1} \cdot\left(\begin{array}{c}
n \\
t
\end{array}\right) \cdot(t+1) ! \cdot(n-t-1) !,
$$

that is, $|D|=K \cdot n ! /(n-t)$ where $K=(t+1) !^{2 t+1}$. With $t$ fixed, we see that

$$
\lim _{n \rightarrow \infty} \frac{|D|}{n !}=K \cdot \lim _{n \rightarrow \infty} \frac{1}{n-t}=0 .
$$

\section{A split basis for the association scheme of the symmetric group}

We now turn our attention to the association scheme of the symmetric group. The center $\mathbb{A}$ of the group algebra of $S_{n}$ is a vector space of symmetric matrices closed under both ordinary matrix multiplication and under Schur (entrywise) multiplication and containing both $I$ and the all-ones matrix $J$. In other words, $\mathbb{A}$ is a Bose-Mesner algebra. The underlying association scheme has $S_{n}$ as its vertex set and contains one graph $\Gamma_{\alpha}$ for each conjugacy class $\mathcal{C}_{\alpha}$ : two permutations $g$ and $h$ are adjacent in $\Gamma_{\alpha}$ if $g h^{-1} \in \mathcal{C}_{\alpha}$. The adjacency matrix of $\Gamma_{\alpha}$ is the matrix $A_{\alpha}$ introduced in Section 3. The basis of primitive central idempotents $E_{\mu}$ was introduced earlier as well.

In the study of association schemes, one often chooses a base point from the vertex set and considers the trivial module $\mathbb{A} \mathbf{e}$ where $\mathbf{e}$ is the elementary basis vector associated to the base point. If we choose the identity permutation as our base point and use the corresponding elementary basis vector $\mathbf{e}_{(1)}$, then the trivial module $\mathbb{A} \mathbf{e}_{(1)}$ for $\mathbb{A}$ acting on $\mathbb{R}^{n !}$ is simply the vector space of class functions. The trivial module has two distinguished bases: the indicator functions $A_{\alpha} \mathbf{e}_{(1)}$ for the conjugacy classes $\mathcal{C}_{\alpha}$ and the irreducible characters $\chi_{\mu}=\frac{n !}{f_{\mu}} E_{\mu} \mathbf{e}_{(1)}$. We found earlier that this module alone was sufficient for the study of $\lambda$-transitive groups. Yet, to extend the results to arbitrary sets of permutations, we found it necessary to appeal to the coding theorist's inner distribution and dual distribution. This is, in effect, a smoothing technique in which we average over all base points $g \in S_{n}$ in order to account for the possible asymmetry of a set $D$.

Consider the set $\mathcal{P}$ of all cosets of Young subgroups of $S_{n}$ partially ordered by inclusion. We partition the elements of this poset into fibers corresponding to the shape of the coset $g Y_{P}$. The matrix $Y_{\lambda}$ introduced in Section 1 is then the incidence matrix between the minimal elements (singletons) of this poset and the elements in the fiber indexed by $\lambda$. We are interested in the matrices $C_{\lambda}=Y_{\lambda} Y_{\lambda}^{\top}$ where $\lambda$ is a partition of $n$. 
Theorem 10. The set $\left\{C_{\lambda}:|\lambda|=n\right\}$ is a basis for $\mathbb{A}$. The change-of-basis equations with respect to the $A_{\alpha}$ have the form

$$
C_{\lambda}=\sum_{\alpha \unlhd \lambda} m_{\lambda, \alpha} A_{\alpha}
$$

with $m_{\lambda, \lambda}>0$. The change-of-basis equations with respect to the $E_{\mu}$ have the form

$$
C_{\lambda}=\sum_{\mu \unlhd \lambda} n_{\lambda, \mu} E_{\mu}
$$

with $n_{\lambda, \mu} \neq 0$ for all $\mu \unlhd \lambda$.

Proof. Since the entry of $C_{\lambda}$ in row $a$ column $b$ is equal to the number of cosets $h Y_{P}$ of Young subgroups of shape $\lambda$ containing both $a$ and $b$, we clearly have $A(g)^{-1} C_{\lambda} A(g)=C_{\lambda}$ for any $g \in S_{n}$ showing that $C_{\lambda}$ lies in $\mathbb{A}$. Next, note that the column space of $C_{\lambda}$ is equal to that of $Y_{\lambda}$ so that, by Corollary 1, $n_{\lambda, \mu} \neq 0$ if $\mu \unrhd \lambda$ and $n_{\lambda, \mu}=0$ otherwise. So the matrices $C_{\lambda}$ indeed form a basis for $\mathbb{A}$.

Now consider the coefficients $m_{\lambda, \alpha}$. The $(a, b)$-entry of $C_{\lambda}$ is non-zero if and only if there is some coset of some Young subgroup of shape $\lambda$ which contains both $a$ and $b$. Equivalently, $a b^{-1}$ is an element of that Young subgroup. But the conjugacy classes $\mathcal{C}_{\alpha}$ which intersect a Young subgroup of shape $\lambda$ are precisely those for which $\alpha$ is a refinement of $\lambda$. So by definition of $A_{\alpha}, m_{\lambda, \alpha} \geq 0$ with strict inequality precisely when $\alpha$ refines $\lambda$. The Theorem now follows since refinement implies $\alpha \unlhd \lambda$ and because $\lambda$ refines itself.

Following Terwilliger [8,16,17], we call the basis $\left\{C_{\lambda}: \lambda\right\}$ a split basis for the association scheme of $S_{n}$. We remark, however, that the typical linear ordering on relations and primitive idempotents is replaced in this case by the partial order $\unlhd$.

In the language of association schemes, a $\lambda$-transitive set $D$ is the same as a Delsarte $\mathcal{T}$-design [6, p32] where $\mathcal{T}=\{\mu: \mu \unrhd \lambda\}$. This provides another viewpoint for our investigation and suggests an avenue for finding future nonexistence results. We remark that Delsarte's linear programming bound — the most obvious technique in this regard - is mostly ineffective in this particular setting, being overshadowed by the following more elementary divisibility conditions on $\lambda$-transitive sets.

Proposition 3. If $D \subseteq S_{n}$ is a $\lambda$-transitive set of permutations, then $|D|$ is divisible by $\left(\begin{array}{l}n \\ \mu\end{array}\right):=\left(\begin{array}{c}n \\ \mu_{1}, \ldots, \mu_{k}\end{array}\right)$ for any partition $\mu \unrhd \lambda$.

Proof. Since $D$ must also be $\mu$-transitive by Theorem 5 , each coset of each Young subgroup of shape $\mu$ contains $r_{\mu}$ permutations from $D$ for some integer $r_{\mu}$. Given a fixed $Y_{P}$ of shape $\mu$, it has $\left(\begin{array}{l}n \\ \mu\end{array}\right)$ cosets which partition $S_{n}$ and thus $D$. So $|D|=r_{\mu}\left(\begin{array}{l}n \\ \mu\end{array}\right)$. 


\section{Open questions}

We list here some open questions raised by our work in the hopes that the reader will be tempted to work on them.

(1) An interesting construction is given in 四 for a set of $13 ! / 7$ ! permutation in $S_{13}$ which Conway calls $M_{13}$. While this set is six-transitive in the sense of [4], it is not $(7,1,1,1,1,1,1)$-transitive in the sense we are using in this paper. We were unable to compute the full degree of transitivity of this set due to its size. We also ask if $M_{13}$ can be combined with some small collection of its translates to yield a 6 -transitive set of permutations in $S_{13}$.

(2) Is there an analogue of $\lambda$-transitive groups and sets for other Coxeter groups? In particular, it would be interesting to investigate the hyperoctahedral group. One would hope that there is a generalization of Theorem 5 in this setting.

(3) In view of Proposition 3, no $\lambda$-transitive subset of $S_{n}$ can contain less than $\left(\begin{array}{l}n \\ \lambda\end{array}\right)$ permutations. Trivial examples where this lower bound is achieved are the cyclic, alternating and symmetric groups. At the end of Section 5, we presented examples achieving this lower bound for $\lambda=(q-2,2)$ where $q$ is an odd prime power. We ask if there are any other infinite families of examples achieving this lower bound.

(4) The Krein parameters $q_{\lambda, \mu}^{\nu}$ of the association scheme of the symmetric group are defined by the equations

$$
E_{\lambda} \circ E_{\mu}=\frac{1}{n !} \sum_{\nu} q_{\lambda, \mu}^{\nu} E_{\nu}
$$

where $\circ$ denotes the entrywise product of matrices. In the study of association schemes, one is often interested in determining which Krein parameters vanish for a given family of schemes. While this question is known to be hard in general, we identify one interesting result along these lines. Using Theorem 2.9.22 on p99 of the book by James and Kerber [4], we find that the Krein parameter $q_{\lambda, \mu}^{\nu}$ vanishes if $\mathrm{d}(\nu)>\mathrm{d}(\lambda)+\mathrm{d}(\mu)$ where $\mathrm{d}(\nu)=\nu_{2}+\nu_{3}+\cdots$.

\section{Acknowledgments}

The authors wish to thank Peter Cameron, Jon Hall, Robert Liebler, Spyros Magliveras and Paul Terwilliger for helpful comments. This paper was written while the first author was visiting the Department of Combinatorics and Optimization at the University of Waterloo. He extends thanks to the department for its hospitality and accommodation during this visit. WJM's research is supported by the Canadian government through NSERC grant number OGP0155422. Additional support was provided by MITACS, by CITO through the Centre for Applied Cryptographic Research, and through NSF-ITR grant number 0112889.

\section{References}


1. E. Bannai and T. Ito. Algebraic Combinatorics I: Association Schemes. Benjamin-Cummings Lecture Note Series 58, The Benjamin-Cummings Publishing Company, Inc., London, 1984.

2. J. Bierbrauer and Y. Edel. Theory of perpendicular arrays. J. Combin. Designs 2 (1994), 375-406.

3. C. J. Colbourn and J. H. Dinitz (eds.). The CRC Handbook of Combinatorial Designs. CRC Press, Boca Raton, 1996.

4. J. H. Conway. M13. pp. 1-11 in Surveys in combinatorics, 1997. London Math. Soc. Lecture Note Ser., 241, Cambridge Univ. Press, Cambridge, 1997.

5. B. A. Davey and H. A. Priestley. Introduction to Lattices and Order. Cambridge Univ. Press, London, 1990.

6. P. Delsarte. An algebraic approach to the association schemes of coding theory. Philips Res. Reports Suppl. 10 (1973).

7. J. D. Dixon and B. Mortimer. Permutation groups. Graduate Texts in Mathematics, 163. Springer-Verlag, New York, 1996.

8. T. Ito, K. Tanabe, and P. M. Terwilliger. Some algebra related to $P$ - and $Q$ polynomial association schemes. pp. 167-192 in Codes and Association Schemes. A. Barg and S. Litsyn eds., DIMACS Ser. Discrete Math. Theoret. Comput. Sci. 56, Amer. Math. Soc., Providence, RI, 2001.

9. G. James and A. Kerber. The Representation Theory of the Symmetric Group. Encyclopedia of Mathematics and Its Applications, 16 . Addison-Wesley, Reading, Mass., 1981.

10. W. M. Kantor. $k$-homogeneous groups. Math. Z. 124 (1972), 261-265.

11. R. A. Liebler and M. R. Vitale. Ordering the partition characters of the symmetric group. J. Algebra 25 (1973), 487-489.

12. D. Livingstone and A. Wagner. Transitivity of finite permutation groups on unordered sets. Math. Z. 90 (1965), 393-403.

13. P. M. Neumann. Generosity and characters of multiply transitive permutation groups. Proc. London Math. Soc. (3) 31 (1975), 457-481.

14. B. E. Sagan. The symmetric group. Representations, combinatorial algorithms, and symmetric functions. (2nd ed.) Graduate Texts in Mathematics, 203. Springer-Verlag, New York, 2001.

15. L. Teirlinck. Non-trivial $t$-designs exist for all $t$. Discrete Math. 65 (1987), 345356.

16. P. M. Terwilliger. Two linear transformations each tridiagonal with respect to an eigenbasis of the other. Linear Algebra Appl. 330 (2001), no. 1-3, 149-203.

17. P. M. Terwilliger. Leonard pairs from 24 points of view. in Proceedings of the Conference: Special Functions 2000 Tempe Arizona, 2000, to appear.

18. D. White. Monotonicity and unimodality of the pattern inventory. Adv. in Math. 38 (1980), 101-108. 